\title{
Perfil dermatoglífico, somatotípico e de composição corporal de judocas brasileiras de alto rendimento
}

Artigo Original

\author{
Mariana Vieira de Melo \\ Programa Stricto Sensu em Ciência da Motricidade Humana da Universidade Castelo \\ Branco - Brasil \\ mvmello@centroin.com.br
}

\author{
José Fernandes Filho \\ Programa Stricto Sensu em Ciência da Motricidade Humana da Universidade Castelo \\ Branco - Brasil
}

jff@cobrase.com.br

MELLO, M.V.; FILHO, J.F. Perfil dermatoglífico, somatotípico e de composição corporal de judocas brasileiras de alto rendimento.

Fitness \& Performance Journal, v.3, n.6, p. 340-349, 2004.

RESUMO: O objetivo deste estudo foi identificar o perfil de composição corporal, somatotípico e dermatoglífico de judocas brasileiras de alto rendimento. Atualmente, milhões de pessoas praticam judô em todo o mundo, seja com propósitos de recreação, manutenção da saúde ou rendimento competitivo nos mais variados níveis. A modalidade foi criada no Japão, em 1882, mas começou a ser praticada pelas mulheres muitos anos depois. Apenas em 1980 aconteceu o primeiro Campeonato Mundial. Por ser o judô feminino uma modalidade muito recente no cenário esportivo mundial, poucos estudos foram realizados nesta área. Este estudo caracterizou-se como uma pesquisa descritiva, que pode ser classificada como tipo perfil e ainda ex post facto. A amostra utilizada constou de 28 atletas $(n=28)$, sendo quatro por categoria de peso, todas ranqueadas pela Confederação Brasileira de Judô entre as seis melhores de cada categoria no país, na classe sênior feminino. Para a análise dos resultados, o estudo observou as condições básicas no tratamento estatístico para manutenção da cientificidade da pesquisa. Há uma lacuna quando se revisa a literatura buscando pelo perfil de atletas de judô brasileiros, principalmente de atletas do sexo feminino de níveis nacional e internacional. O objeto teórico e formal desta pesquisa está então centrado na atleta de judô feminino de alto rendimento, mas com a intencionalidade de que os resultados aqui obtidos possam influenciar na compreensão fenomenológica e axiológica dos aspectos motrizes do Ser Praticante do desporto, em todos os seus níveis de complexidade. O presente trabalho insere-se no Programa de Pós-Graduação em Ciência da Motricidade Humana, com enfoque Biofísico da Motricidade Humana, na área Temática de Treinamento da Performance Motora e de Alto Rendimento, dentro da linha de pesquisa Somatotipia e Dermatoglifia da Performance Motora. Para análise dos resultados consideraram-se primeiramente as categorias de peso de forma individual, com quatro sujeitos em cada uma. Posteriormente, analisaram-se os resultados considerando quatro grupos, formados por cada duas categorias de peso, em ordem crescente, sendo que a categoria pesado formou sozinha o quarto grupo. Considerou-se ainda uma média geral, relacionada ao grupo como um todo, constando de 28 indivíduos (amostra completa). As variáveis analisadas apresentaram as seguintes médias e desvios padrões, para o grupo como um todo $(\mathrm{n}=28)$ : idade de 23,3 anos $\pm 3,8$; estatura de $164,8 \mathrm{~cm} \pm 1,4 ;$ massa corporal de $69,4 \mathrm{~kg} \pm 4,2 ;$ percentual de gordura de $20,1 \% \pm 8,7$; endomorfia de $3,6 \pm 1,9$; mesomorfia de $5,1 \pm 1,7$; ectomorfia de $1,5 \pm 0,9$; desenho tipo arco (A) de $0,6 \pm 1,1$; desenho tipo presilha (L) de $6,3 \pm 2,7$; desenho tipo verticilo (W) de 3,2 $\pm 3,0$; delta 10 (D10) de 12,6 $\pm 3,6$; somatório da quantidade total de linhas de 109,1 $\pm 34,4$. Com relação às fórmulas digitais, encontrou-se o seguinte resultado percentual médio: 10 A de $0,0 \%$; AL de 10,7\%; ALW de 17,9\%; 10L de 7,1\%; L=W de 10,7\%; 10W de 7,1\%; L >W de 35,7\% e W $>$ L de $10,7 \%$. Com base nos resultados apresentados, concluiu-se que este estudo respondeu ao problema proposto, encontrando um perfil das atletas de judô feminino de alto rendimento no Brasil, para as variáveis propostas.

Palavras-chave: Judô, perfil, composição corporal, somatotipo, dermatoglifia.

Endereço para correspondência:

Rua Oscarito, 155 - Freguesia - Rio de Janeiro - RJ - CEP 22743-730

Data de Recebimento: outubro / 2004

Data de Aprovação: novembro / 2004

Copyright@ 2008 por Colégio Brasileiro de Atividade Física, Saúde e Esporte.

6


The dermatoglyphic, somatotypical and body composition profile of high level female judo athletes

The aim of this study was to identify body composition, somatotype and dermatoglyphic profiles of high performance Brazilian female judo athletes. Nowadays, millions of people practice judo around the world, for recreation, health maintenance or competition performance on different levels. The sport was created in Japan, 1882, but women started playing it many years after. The first Women World Championship happened only in 1980. Because female judo is a very recent condition in the sports scenery, only a few studies were made about it. This study was characterized as a descriptive research, classified as profile and ex post facto study. The sample was composed by 28 athletes $(n=28)$, four in each weight category; all of them included in the six best athletes of each category in the Brazilian Federation women ranking. For results analysis, the study observed the basic conditions in statistics for research scientificity. There is a gap when we look for references on Brazilian judo athletes' profiles, particularly female athletes of national and international levels. The theoretical and formal object of this research is centered in the high performance female judo athlete, but with intentionality that the results found here could influence the phenomenological and axiological comprehension of the athletes ' motor aspects, in all complexity levels. The present research is included in the Human Motor Science Graduation Program with emphasis on Biophysics, in the semantic area of Motor Performance Training. To analyze the results it was first considered the weight categories individually, with four subjects in each of these categories. After that, the results were analyzed considering four groups, formed by two weight categories each one, in a crescent order. The heaviest weight category was analyzed alone forming a separated forth group. It was also considered a total mean, related to the total sample with 28 subjects. The variables analyzed displayedthe following means and standard deviations for the total sample: age 23,3 years $\pm 3,8$; stature 164,8 $\mathrm{cm} \pm 1,4$; body mass $69,4 \mathrm{~kg} \pm 4,2$; body fat $20,1 \% \pm 8,7$; endomorph 3,6 $\pm 1,9$; mesomorph $5,1 \pm 1,7$; ectomorph $1,5 \pm 0,9$; arch draw type (A), 0,6 $\pm 1,1$; loop draw type (L), 6,3 $\pm 2,7$; whorl draw type (W), 3,2 $\pm 3,0$; D10 $12,6 \pm 3,6 ;$ SQTL 109,1 $\pm 34,4$. Relating to the digital formulas, it was found the following mean percent results: 10A, 0,0\%; AL, 10,7\%; ALW, 17,9\%; 10L, $7,1 \% ; L=W, 10,7 \% ; 10 W, 7,1 \% ; L>W, 35,7 \%$ and $W>L, 10,7 \%$. Based on these results, it was concluded that this research answered the proposed problem, finding the profile of Brazilian senior female judo athletes in high performance, for the proposed variables.

Keywords: Judo profile, body composition, somatotype, dermatoglyph
Perfil dermatoglifico, composicion corporal y somatotipico de judocas brasileñas de alto rendimento

El objetivo de éste estudio fue identificar el perfil de composición corporal, somatotípico y dermatog lífico de judocas brasileñas de alto rendimiento. Actualmente, millones de personas practican al judo en todo el mundo, con propósitos de recreación y manutención de la salud, o para buscar el rendimiento competitivo en los más variados niveles. La modalidad fue creada en Japón en 1882, pero empezó a ser practicada por las mujeres muchos años después. Solamente en 1980 ocurrió el primer Campeonato Mundial. Por ser el judo femenino una modalidad muy reciente en el escenario deportivo mundial, pocos estudios fueron realizados en ésta área. Éste estudio se caracterizó como una investigación descriptiva, que puede ser clasificada como tipo perfil y aún ex post facto. La muestra utilizada constó de 28 atletas $(n=28)$, siendo cuatro por categoría de peso, todas ranqueadas por la Confederación Brasileña de Judo entre las seis mejores de cada categoría en el país, en la clase senior femenino. Para el análisis de los resultados, el estudio observó las condiciones básicas en el tratamiento estadístico para manutención de la cientificidad de la investigación. Hay un vacío cuando uno revisa la literatura buscando el perfil de atletas de judo brasileños, principalmente de atletas del sexo femenino de niveles nacional e internacional. Para el análisis de los resultados fue considerado primeramente, las categorías de peso individualmente, con cuatro sujetos en cada una. Posteriormente fueron analizados los resultados considerando cuatro grupos, formados por cada dos categorías de peso, en orden creciente, siendo que la categoría pesado formó sóla el cuarto grupo. Se consideró aún, una media general, relacionada al grupo total, constando de 28 individuos (muestra completa). Las variables analizadas presentaron las siguientes medias y desviación típica, para el grupo total $(n=28)$ : edad de 23,3 años $\pm 3,8$; talla de $164,8 \mathrm{~cm} \pm 1,4$; masa corporal de 69,4 $\mathrm{kg} \pm 4,2 ;$ porcentual de grasa de $20,1 \% \pm 8,7$; endomorfia de $3,6 \pm 1,9 ;$ mesomorfia de 5,1 $\pm 1,7$; ectomorfia de 1,5 $\pm 0,9$; diseño tipo arco (A) de $0,6 \pm 1,1$; diseño tipo presilla $(L)$ de $6,3 \pm 2,7$; diseño tipo verticilo $(W)$ de 3,2 $\pm 3,0$; delta 10 (D10) de 12,6 $\pm 3,6$; la suma de la cantidad total de líneas de 109,1 $\pm 34,4$. Con relación a las fórmulas digitales, se encontró como resultado porcentual medio: 10A de 0,0\%; AL de 10,7\%; ALW de 17,9\%; $10 \mathrm{~L}$ de 7,1\%; $L=W$ de $10,7 \%$; $10 \mathrm{~W}$ de $7,1 \%$; $\mathrm{L}>\mathrm{W}$ de $35,7 \%$ y $\mathrm{W}>\mathrm{L}$ de $10,7 \%$. Con base en los resultados presentados, se concluyó que éste estudio respondió al problema propuesto, encontrando un perfil de las atletas de judo femenino de alto rendimiento en Brasil, para las variables propuestas.

Palabras clave: Judo, perfil, composición corporal, somatotipo, dermatoglifia

\section{INTRODUCÃO}

Atualmente, milhões de pessoas em todo o mundo praticam judô, sejam com propósitos de recreação, manutenção da saúde ou com objetivos de rendimento competitivo nos mais diversos níveis. A popularidade mundial do esporte é evidenciada pelos mais de cem países filiados à Federação Internacional de Judô (FIJ) e pelos inúmeros campeonatos internacionais que ocorrem todos os anos. Apesar de seu pouco tempo de história (foi criado no Japão em 1882), o Judô, segundo Franchini (1999), já é a modalidade de luta mais praticada no Brasil, tendo aproximadamente dois milhões de praticantes, o dobro do número de adeptos do karatê, sendo ainda a segunda modalidade geral em número de praticantes. A qualidade técnica do judô brasileiro está entre as melhores do mundo. Os resultados conquistados pela equipe brasileira nos últimos Jogos Olímpicos e Competições Internacio- nais comprovam este fato (GRECO; VIANA, 1997). Mas, apesar disto, o judô ainda tem uma bibliografia especializada bastante deficiente. Estudos com atletas de judô feminino são ainda mais difíceis de serem encontrados.

○ judô começou a ser praticado pelas mulheres muitos anos após ter sido criado, em 1882. Somente em 1980 o judô feminino veio a ter seu primeiro Campeonato Mundial, na cidade de Nova York. $\bigcirc$ judô feminino só foi incluído como competição oficial nos Jogos Olímpicos de Barcelona, em 1992.

É de suma importância para pesquisadores e, principalmente, treinadores conhecer as características das judocas de alto rendimento no país, para que possam elaborar treinamentos mais eficazes para desenvolver as habilidades necessárias ao 
desporto e identificar em suas atletas, comparando-as com as de alto rendimento, as maiores deficiências, corrigindo-as com mais eficácia. Outro importante motivo para se traçar um perfil de judocas competidoras adultas, utilizando-se uma amostra representativa da população, é identificar as diferenças entre as atletas de alto rendimento e as de rendimento médio, possibilitando relacionar os componentes necessários para uma futura seleção de talentos no judô.

objetivo principal deste estudo foi identificar os perfis dermatoglífico, somatotípico e de composição corporal de judocas brasileiras de alto rendimento, competidoras da classe sênior (adulto). A identificação de um perfil destas atletas poderia servir de base para uma futura seleção de talentos para o judô feminino brasileiro.

A dermatoglifia - do latim dermo, significando "pele" e do grego glypha, "gravar" - é um termo proposto por Cummins e Midlo (1942), em 1926. Fernandes Filho (2003a) cita que, devido a características como a imutabilidade, inalterabilidade e imitabilidade, a dermatoglifia ganhou muita importância, principalmente nas áreas de Medicina Legal, Criminalística e Antropologia Física.

Os padrões dermatoglíficos são estabelecidos por volta do terceiro a quarto mês de vida fetal e permanecem estáveis com a idade, fazendo com que o desenvolvimento pós-natal não tenha qualquer papel na variabilidade dermatoglífica, exceto em algumas condições patológicas, trazendo vantagem sobre outras medidas físicas ou fisiológicas em humanos (Nikitiuk, 1988 citado por JOÃO; FERNANDES FILHO, 2002).

Os parâmetros da dermatoglifia dos dedos mais simples e acessíveis para o reconhecimento são os desenhos nas falanges distais dos dedos. A estrutura do relevo cutâneo dos dedos é bem estudada. Segundo Pável (2003), a maioria dos autores distingue três grupos de desenhos: arco, presilha e verticilo. A forma dos desenhos constitui uma característica qualitativa, sendo que a quantidade de linhas $(Q L)$, o somatório da quantidade total de linhas (SQTL) e a quantidade de cristas cutâneas dentro do desenho representam características quantitativas. Segundo Silva et al. (2003), a avaliação da intensidade de desenhos se efetua partindo da presença dos deltas, e se calcula, assim, o chamado índice de deltas (D10). O Arco (A) é um desenho que não possui deltas; a Presilha (L) - o desenho de um delta; o Verticilo (W) - o desenho de dois deltas. Cada tipo de desenho recebe uma avaliação correspondente - 0, 1 e 2, respectivamente para A, L e W. Ou seja, a avaliação máxima é 20 e a mínima é 0 (a soma de deltas nos 10 dedos). $O$ desenho mais simples é o arco e o mais complexo é o verticilo (Gladkova, 1966, citada por MACÊDO; FERNANDES FILHO, 2003).

As Impressões Digitais (ID) são estruturas hereditariamente determinadas que têm a multiformidade estrutural, sendo que são diferenciadas filogeneticamente e antropogeneticamente para a execução das funções mecânicas e tácteis complicadas que se distinguem por sua incomparabilidade individual, ou seja, são marcas genéticas universais (FERNANDES FILHO, 1997).
Conforme Fernandes Filho (1997), são de grande interesse as pesquisas realizadas pelo Laboratório de Antropologia, Morfologia e Genética Esportiva do VNIIFK, em Moscou. Estas pesquisas ocorreram a partir de 1966 e se realizavam em duas direções: dermatoglifia digital - as qualidades físicas, e dermatoglifia digital - o tipo de atividade esportiva. $\bigcirc$ mesmo autor afirma que o modelo de ID conduz à escolha mais adequada da especialização esportiva, com a perspectiva de otimização do talento individual.

Nas décadas de 70 e 80, surgiram os trabalhos de Nikitiuk e de seus alunos, dedicados aos problemas das ID na teoria e na prática da seleção esportiva e individualização da preparação (Cavostianova; Coloxko, 1990, citados por FERNANDES FILHO, 1997). Mostrou-se que a complexidade dos desenhos pode servir de marcas de prognóstico da compleição definitiva. Nikitiuk, Abramova e Ozolin (citados por FERNANDES FILHO, 2003) pesquisaram um esquema de princípios de associação das impressões digitais com as qualidades físicas. Segundo Silva et al. (2003), a velocidade e a força explosiva são caracterizadas pelo aumento da quantidade de presilhas $(L>7)$, diminuição dos verticilos $(\mathrm{W}<3)$, presença e aumento dos arcos, e a redução do SQTL. Já a capacidade aeróbia e as atividades de combinações motoras complexas são caracterizadas pela diminuição dos arcos (até 0 ) e presilhas $(L<6)$, aumento dos verticilos $(W>4)$ e aumento do SQTL. Portanto, o aumento da quantidade de linhas é contrário ao desenvolvimento das qualidades de velocidade e de força (GUBA; TCHERNOVA, 1995, citados por FERNANDES FILHO, 1997). A estabilidade estática está correlacionada com a baixa quantidade de linhas e com a baixa intensidade de desenhos (ARUTIOHIAN, 1988, citado por FERNANDES FILHO, 1997). Detectou-se a correlação de componentes da memória motora com o tipo dos desenhos: componente visual - presilhas, e componente proprioceptivo-motor - verticilos (COLOBEBA; TCHERKAZOV, 1988, citados por FERNANDES FILHO, 1997). Comprovou-se a ligação da quantidade de linhas com o VO2 máximo, mas apenas nos grupos femininos, refletindo indiretamente a correlação da complexidade de desenhos com a resistência (CHUARTZ; ALEKCEEV, 1988, citados por FERNANDES FILHO, 1997).

Fernandes Filho (1997) afirma, ainda, que o modelo de ID conduz à escolha mais adequada da especialização esportiva, com a perspectiva de otimização do talento individual. Esta metodologia de marcas genéticas se evidenciaria para a otimização de um processo, aumentando a certeza de manterem-se sob a mira indivíduos que tenham uma predisposição genética para este ou aquele tipo de modalidade esportiva. As modalidades

\section{Figura 1 - Tipos de desenhos nas impressões digitais}

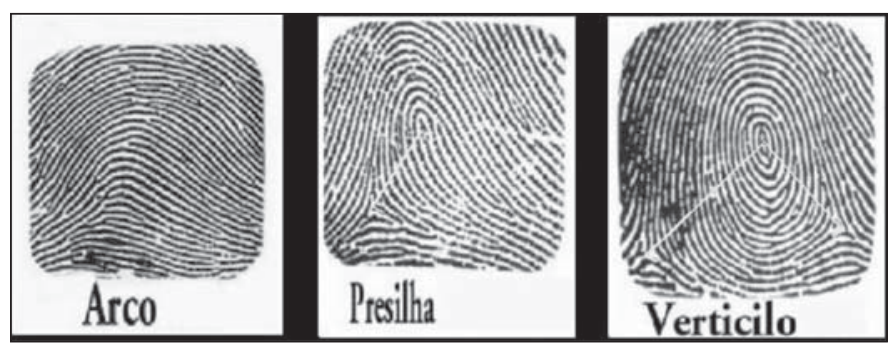


de esporte de velocidade e de força se inserem no campo de valores baixos de D10 e de SQTL; as modalidades caracterizadas por uma propriocepção complexa, no campo de valores altos; os grupos de esportes de resistência, a posição intermediária. Fernandes Filho et al.(2004) afirmam, ainda, que a dermatoglifia é um marcador genético de amplo espectro para utilização em associação com as qualidades físicas básicas e a tipologia de fibras musculares.

\section{METODOLOGIA}

Gráfico 1 - Médias de \% de gordura corporal por categoria de peso e geral

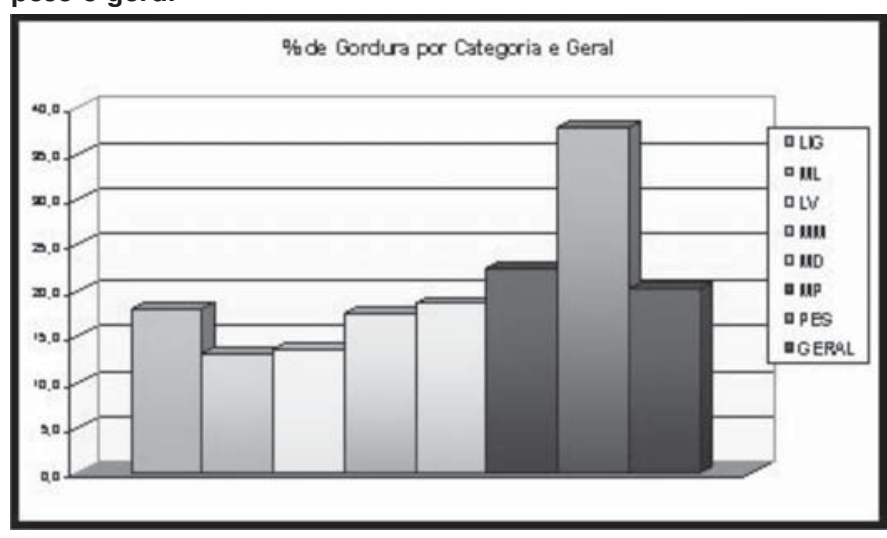

Gráfico 3 - Médias de somatotipo por categoria de peso e geral

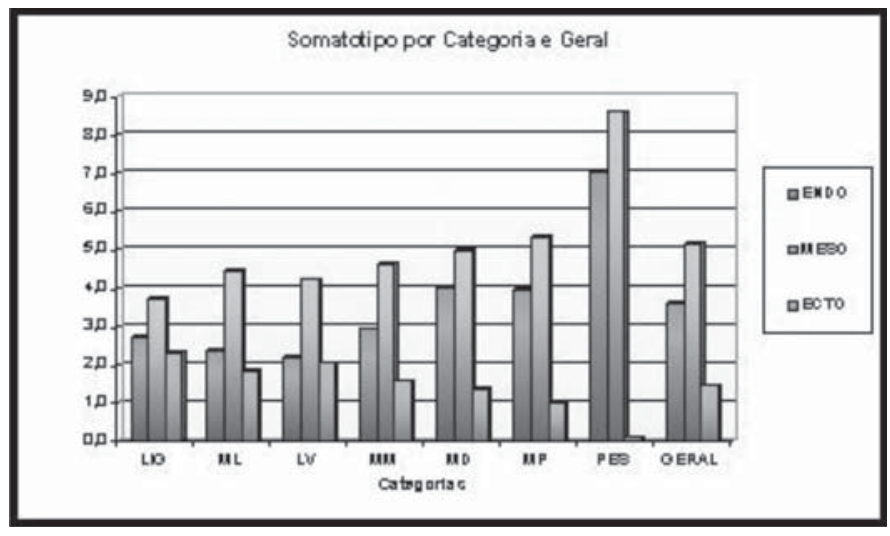

Gráfico 5 - Médias de tipo de desenho por categoria de peso e geral

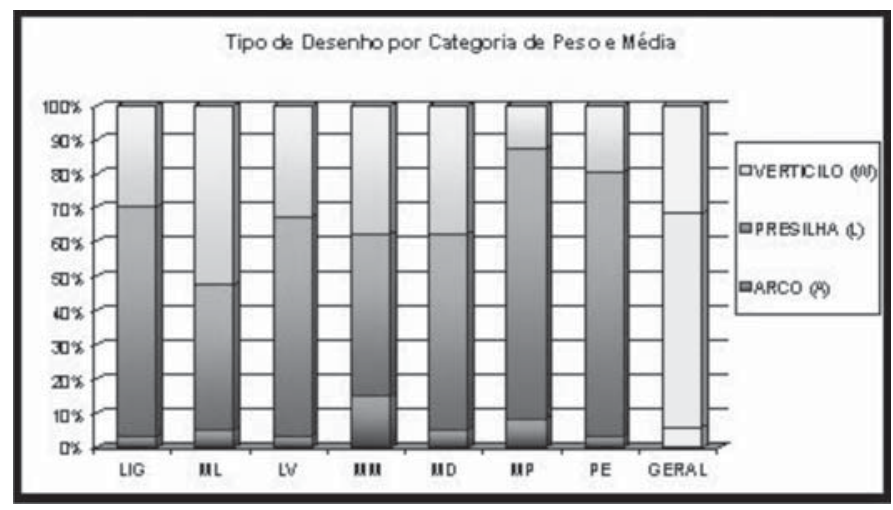

presente estudo é uma pesquisa descritiva, podendo ser classificada como tipo perfil ou ainda ex post facto. Todos os sujeitos selecionados para este estudo estavam entre as seis melhores atletas de judô feminino sênior do país em cada uma das sete categorias de peso, de acordo com o ranking oficial da Confederação Brasileira de Judô, totalizando uma amostra de 28 atletas, quatro por categoria de peso. Esta pesquisa seguiu rigorosamente os critérios propostos pela resolução n 196, de 10 de outubro de 1996, do Conselho Nacional de Saúde, e as atletas foram voluntárias para o estudo. Utilizou-se, para caracterização do perfil de composição corporal, a equação de Jackson et al. (1980) para estimar a densidade corporal. Para

Gráfico 2 - Médias de \% de gordura corporal por grupo e geral

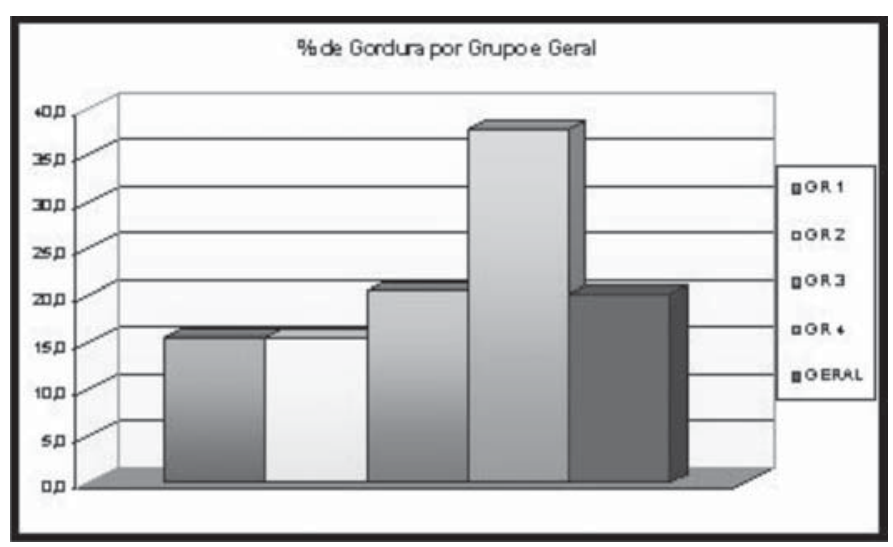

Gráfico 4 - Médias de somatotipo por grupo e geral

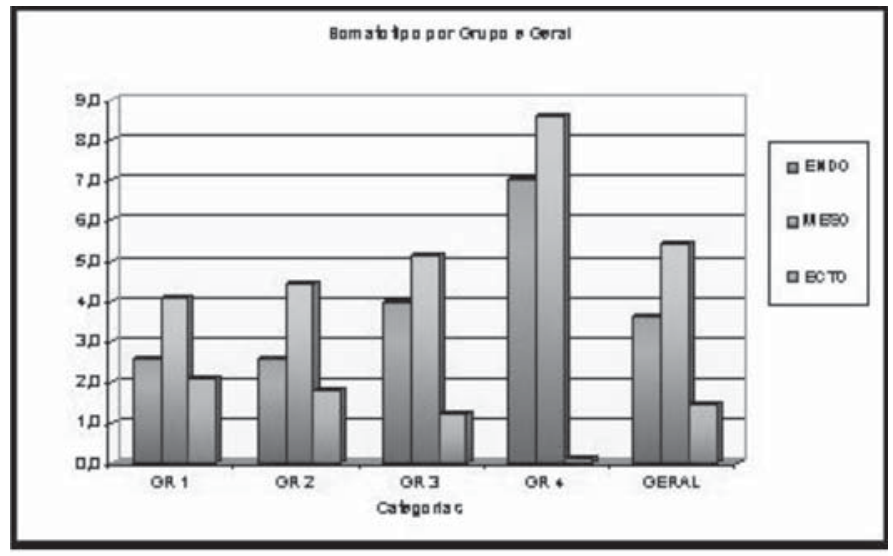

Gráfico 6 - Médias de tipo de desenho por grupo e geral

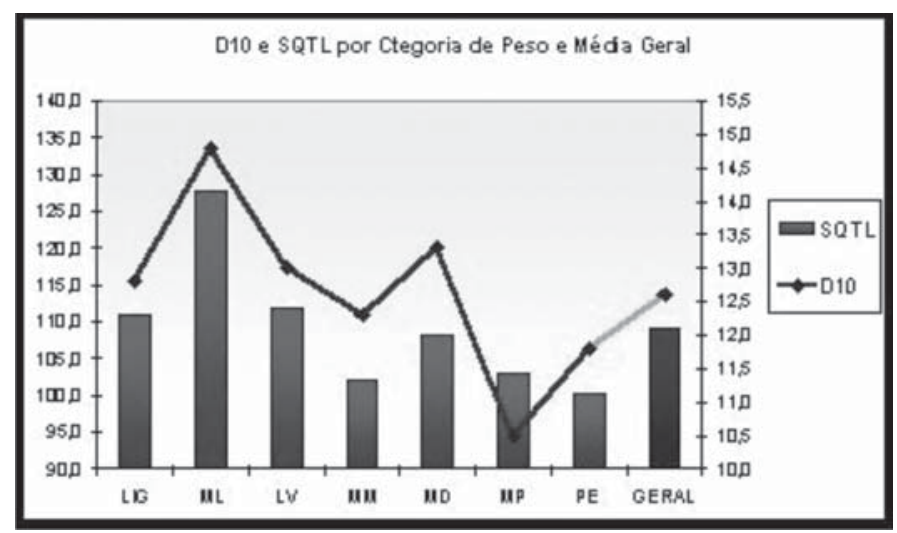




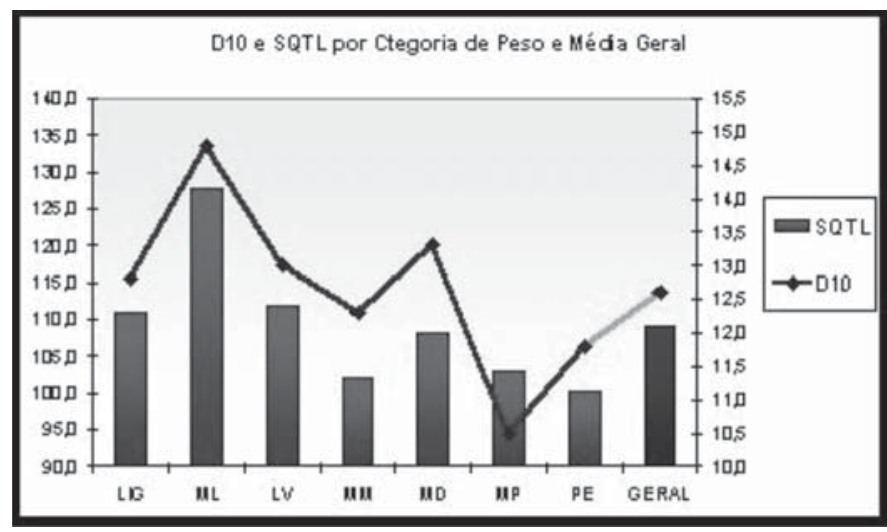

Gráfico 9 - Distribuição das fórmulas digitais para a média geral da amostra

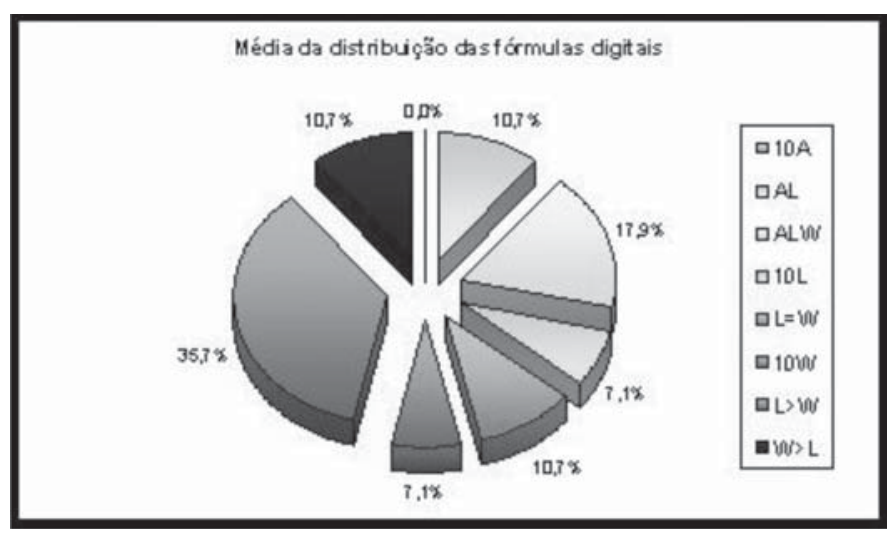

o cálculo do percentual de gordura utilizou-se a equação de Siri (1961). Para o somatotipo utilizou-se o protocolo de Heath e Carter, que possui, segundo Carter e Heath (1990), um $r=$ 0,98 . As medidas corporais foram tomadas de acordo com o protocolo proposto pela Sociedade Internacional para o Avanço da Cineantropometria (ISAK). Os instrumentos utilizados foram balança da marca Filizola, compasso da marca Harpender, paquímetro da marca Rosscraft, coletor de impressões digitais da marca Impress.

Para a análise das impressões dermatoglíficas, foi utilizado o protocolo de Cummins e Midlo (1942). Após a coleta das impressões digitais, o protocolo preconiza:

1. Identificar os tipos de desenho (figura 1) - $\operatorname{arco}(A)$, presilha (L) ou verticilo (W);

2. Contar a quantidade de linhas em cada dedo da mão (QL);

3. Contar a quantidade de linhas em todos os dez dedos das mãos (SQTL);

4. Determinar a delta 10 (D10) - intensidade sumária dos desenhos;

5. Determinar os tipos de fórmulas digitais.

\section{APRESENTAÇÃo E DISCUSSÃO DOS RESULTADOS}

○ judô apresenta sete categorias de peso, que vão do ligeiro ao pesado. Os dados apresentados foram divididos por categoria de peso, pois as características das atletas apresentam grande
Gráfico 8 - Médias de D10 e SQTL por grupo e geral

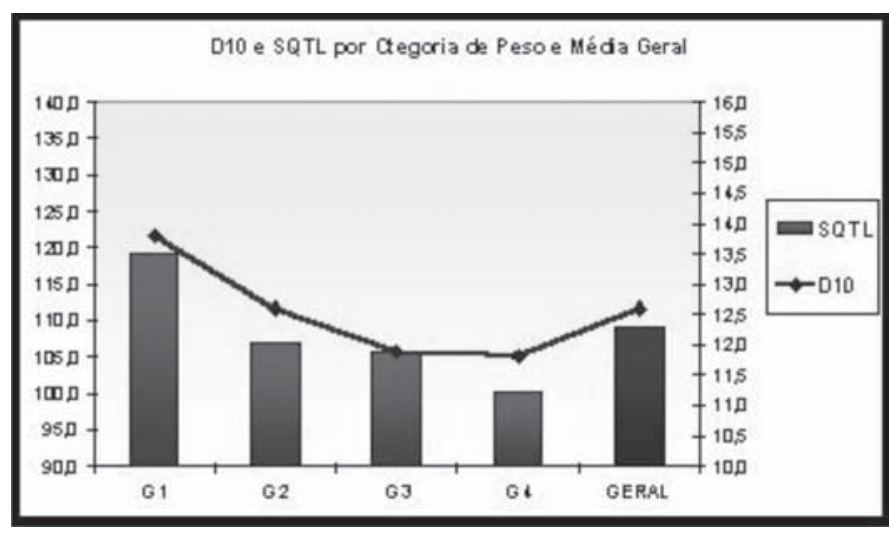

Gráfico 10 - Normatização das variáveis A, L, W, D10 e SQTL

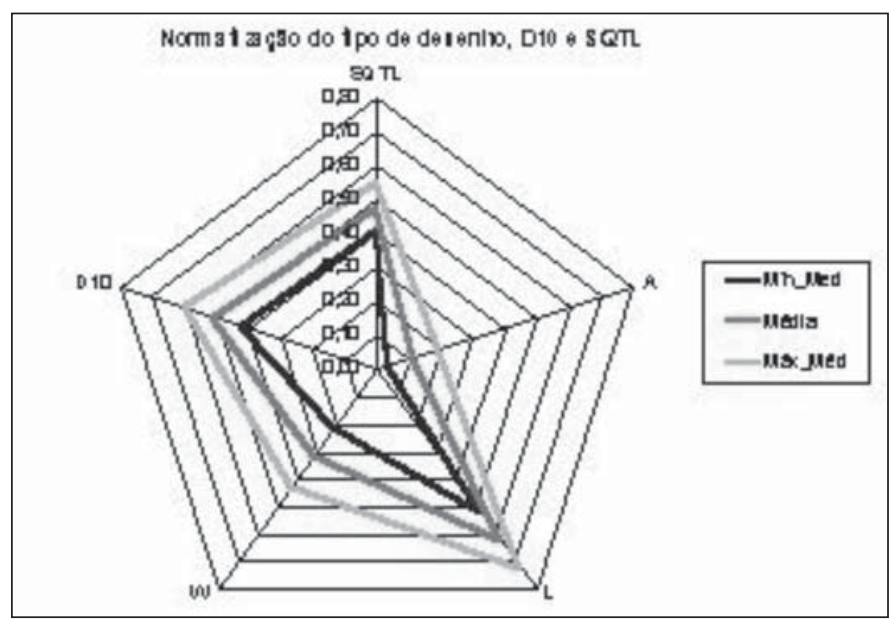

variação, dependendo da categoria à qual pertencem. Os dados estão apresentados também por grupos de categorias, onde o Grupo 1 corresponde às categorias Ligeiro e Meio-leve (massa corporal menor), o Grupo 2 corresponde às categorias Leve e Meio-médio, o Grupo 3 reúne as categorias Médio e Meiopesado e o Grupo 4 corresponde à categoria Pesado (massa corporal maior).

\section{Caracterização da Amostra}

A média de idade das atletas incluídas na amostra, aferidas pelo ano de nascimento, foi de 23,3 \pm 3,8 anos (mínimo de 18,0 e máximo de 29,0$)$. A categoria com menor média é a pesado, com 22,3 anos e a com maior média é a categoria médio, com 24,5 anos.

Para esta amostra foram encontradas estatura média de 164,8 \pm 1,4 cm (máximo de 177,0 e mínimo de 154,0cm) e massa corporal de 69,4 \pm 4,2 kg (mínimo de 50,9 e máximo de $115,0 \mathrm{~kg}$ ). Na tabela 1, podemos observar os valores médios por categoria de peso e geral para a estatura e massa corporal.

Pode-se reparar que, em período de treinamento, todas as médias de massa corporal das categorias estão acima do limite máximo permitido para a competição, com exceção das categorias meio médio $(-63,0 \mathrm{~kg})$ e meio pesado $(-78$ $\mathrm{kg})$. Este fato poderia indicar que a grande maioria das atletas 
Tabela 1 - Médias de estatura e massa corporal por categoria de peso e geral

\begin{tabular}{lcccc}
\hline CATEGORIAS & \multicolumn{2}{c}{ ESTATURA $(\mathrm{cm})$} & \multicolumn{2}{c}{ MASSA CORPORAL $(\mathrm{kg})$} \\
\hline & MÉDIA & $\mathrm{S}$ & MÉDIA & S \\
\hline Ligeiro (-48kg) & 157,3 & $\pm 3,4$ & 52,6 & $\pm 2,2$ \\
Meio Leve (-52kg) & 157,3 & $\pm 3,8$ & 54,4 & $\pm 1,4$ \\
Leve (-57kg) & 164,3 & $\pm 4,0$ & 60,8 & $\pm 2,8$ \\
Meio Médio (-63kg) & 163,8 & $\pm 4,0$ & 63,1 & $\pm 3,3$ \\
Médio (-70kg) & 171,3 & $\pm 4,3$ & 73,5 & $\pm 4,3$ \\
Meio Pesado (-78kg) & 171,8 & $\pm 2,2$ & 103,3 & $\pm 1,4$ \\
Pesado (+78kg) & 168,3 & $\pm 6,9$ & 69,4 & $\pm 13,4$ \\
Média Geral & 164,8 & $\pm 1,4$ & $\pm 4,2$ \\
\hline
\end{tabular}

Tabela 2 - Médias de \% de gordura corporal por categoria de peso e geral

\begin{tabular}{lcc}
\hline CATEGORIAS & \multicolumn{2}{c}{$\%$ DE GORDURA } \\
\hline & MÉDIA & $\mathrm{S}$ \\
Ligeiro (-48kg) & 18,0 & $\pm 3,6$ \\
Meio Leve $(-52 \mathrm{~kg})$ & 13,0 & $\pm 2,6$ \\
Leve $(-57 \mathrm{~kg})$ & 13,7 & $\pm 1,5$ \\
Meio Médio $(-63 \mathrm{~kg})$ & 17,5 & $\pm 4,5$ \\
Médio (-70kg) & 18,6 & $\pm 2,2$ \\
Meio Pesado (-78kg) & 22,4 & $\pm 7,1$ \\
Pesado (+78kg) & 37,8 & $\pm 4,9$ \\
Média Geral & 20,1 & $\pm 8,7$ \\
\hline
\end{tabular}

necessita utilizar algum tipo de recurso de perda de peso no período pré-competitivo. De acordo com os dados coletados, das 24 atletas com limite máximo de peso na categoria (a categoria pesado não tem limite de peso), 18 estava com o peso acima do limite de sua categoria, o que representa $75 \%$ da amostra. Cabe ressaltar que os dados foram coletados 28 dias antes da próxima seletiva, importante para todas as atletas da amostra.
Tabela 3 - Médias de \% de gordura corporal por grupo e geral

\begin{tabular}{lcc}
\hline GRUPOS & \multicolumn{2}{c}{$\%$ de gordura } \\
\hline & MÉDIA & $\mathrm{S}$ \\
Grupo 1 & 15,5 & $\pm 3,9$ \\
Grupo 2 & 15,6 & $\pm 3,7$ \\
Grupo 3 & 20,5 & $\pm 5,3$ \\
Grupo 4 & 37,8 & $\pm 4,9$ \\
Média Geral & 20,1 & $\pm 8,7$ \\
\hline
\end{tabular}

\section{Composição Corporal}

Na tabela 2 e gráfico 1, podemos observar os valores médios por categoria de peso e geral para o percentual de gordura (\%G). Para esta amostra foi encontrado, através das fórmulas de Jackson et al. (1980) e Siri (1961), um percentual de gordura médio de 20,1 \pm 8,7 \% (mínimo de 10,7 e máximo de 42,2\%).

Na tabela 3 e gráfico 2, podemos observar os valores médios por grupo e geral para o \%G corporal.

Ao analisarmos a tabela 3 e gráfico 2, observamos uma clara tendência de aumento de percentual de gordura com o aumento da categoria de peso, com exceção da categoria ligeiro

Tabela 4 - Médias de \% de gordura corporal encontradas em outros estudos.

\begin{tabular}{lccc}
\hline AUTOR & AMOSTRA & N & \%GORD \\
\hline Vidalin et al citado por Franchini (2003) & Francesas & 07 & 21,7 \\
Callister et al. (1990) & Americanas de elite & 07 & 15,8 \\
Callister et al. (1991) & Americanas de elite & 09 & 15,2 \\
Litlle (1991) & Canadenses & 08 & 15,2 \\
Franchini et al. (1996) & Sel. Brasileira Univers. & 07 & 16,1 \\
Franchini et al. (1999) & Seleção Brasileira & 07 & 22,7 \\
Mello at al. (2000) & Seleção do RJ & 08 & 20,3 \\
Moraes e Mello (2001) & Elite Nacional & 28 & 19,5 \\
Mello (2004)- este estudo & Elite Nacional & 28 & 20,1 \\
\hline
\end{tabular}

Tabela 5 - Médias de somatotipo por categoria de peso e geral

\begin{tabular}{lccc}
\hline CATEGORIAS & ENDOMORFIA & MESOMORFIA & ECTOMORFIA \\
\hline & MÉDIA \pm S & MÉDIA S SÉDIA \pm S & $2,3 \pm 0,8$ \\
Ligeiro (-48kg) & $2,7 \pm 0,5$ & $3,7 \pm 0,9$ & $1,8 \pm 0,9$ \\
Meio Leve (-52kg) & $2,3 \pm 0,6$ & $4,4 \pm 0,7$ & $2,0 \pm 0,8$ \\
Leve (-57kg) & $2,2 \pm 0,4$ & $4,2 \pm 1,1$ & $1,6 \pm 0,5$ \\
Meio Médio (-63kg) & $2,9 \pm 0,8$ & $4,6 \pm 0,3$ & $1,4 \pm 0,2$ \\
Médio (-70kg) & $4,0 \pm 1,3$ & $5,0 \pm 0,7$ & $1,0 \pm 0,4$ \\
Meio Pesado (-78kg) & $3,9 \pm 1,8$ & $5,3 \pm 0,8$ & $0,1 \pm 0,0$ \\
Pesado (+78kg) & $7,0 \pm 1,6$ & $8,6 \pm 0,5$ & $1,5 \pm 0,9$ \\
Média Geral & $3,6 \pm 1,9$ & $5,1 \pm 1,7$ & \\
\hline
\end{tabular}


$(-48,0 \mathrm{~kg})$, que tem um percentual de gordura acima da categoria meio leve, da leve e da meio-médio, praticamente igualando-se ao da categoria médio $(-70,0 \mathrm{~kg})$. Se observarmos a tabela 1, veremos que a massa corporal média da categoria ligeiro está acima do limite da categoria seguinte, que vai até $52,0 \mathrm{~kg}$. Isso demonstra que as atletas desta categoria são as atletas que têm maior problema para conseguir controlar seu peso no período de treinamento pré-competitivo. $\bigcirc$ percentual de gordura elevado sugere que, provavelmente, elas não fazem uma dieta adequada em longo prazo, o que seria o ideal. Observamos ainda que a categoria pesado tem uma média de percentual de gordura de $37,8 \%$, quase cinqüenta por cento a mais do que a média da categoria meio pesado, que é de $22,4 \%$. Talvez, se estas atletas diminuíssem o seu percentual de gordura e aumentassem a massa muscular, pudessem ter resultados internacionais mais expressivos. Estudos de composição corporal em atletas de judô do sexo feminino são pouco freqüentes, como afirma Franchini (2001). A tabela 4 mostra os resultados encontrados para percentual de gordura em alguns destes poucos estudos realizados com judocas de alto rendimento.

\section{Somatotipo}

Na tabela 5 e gráfico 3, podemos observar os valores médios por categoria de peso e geral para os três componentes do somatotipo. Para esta amostra foi encontrado, através do sistema de Heath e Carter (CARTER E HEATH, 1990), valores médios de 3,6 - 5,1 - 1,5 para endomorfia, mesomorfia e ectomorfia, respectivamente (mínimo de 1,5 - 2,9 - 0,1, máximo de 9,2 $8,9-3,4)$. Segundo os autores, podemos classificar as atletas de judô como mesomorfas em todas as categorias de peso, por terem o componente mesomorfia dominante e a endomorfia e ectomorfia mais que $1 / 2$ unidade menores.

Tabela 6 - Médias de somatotipo por grupo e geral

\begin{tabular}{lccc}
\hline GRUPOS & ENDOMORFIA & MESOMORFIA & ECTOMORFIA \\
\hline & MÉDIA \pm S & MÉDIA \pm S & MÉDIA \pm S \\
Grupo 1 & $2,5 \pm 0,6$ & $4,1 \pm 0,8$ & $2,1 \pm 0,8$ \\
Grupo 2 & $2,6 \pm 0,7$ & $4,4 \pm 0,8$ & $1,8 \pm 0,7$ \\
Grupo 3 & $4,0 \pm 1,4$ & $5,2 \pm 0,7$ & $1,2 \pm 0,3$ \\
Grupo 4 & $7,0 \pm 1,6$ & $8,6 \pm 0,5$ & $0,1 \pm 0,0$ \\
Média Geral & $3,6 \pm 1,9$ & $5,1 \pm 1,7$ & $1,5 \pm 0,9$ \\
\hline
\end{tabular}

Tabela 7 - Médias de somatotipo em outros estudos

\begin{tabular}{|c|c|c|c|c|c|}
\hline AUTOR & AMOSTRA & $\mathrm{N}$ & ENDO & MESO & ECTO \\
\hline BRIEF (1986, citado por CARTER e HEATH, 1990) & Jogos Bolivarianos & 06 & 4,1 & 4,1 & 1,8 \\
\hline Franchini et al. (1999) & Seleção Brasileira & 07 & 4,3 & 5,1 & 1,1 \\
\hline Franchini et al. (1996) & Sel. Bras. Universitária & 07 & 4,1 & 5,0 & 1,7 \\
\hline Mello at al. (2000) & Seleção do RJ & 08 & 4,1 & 4,8 & 2,1 \\
\hline Mello (2004) - este estudo & Elite brasileira & 28 & 3,6 & 5,1 & 1,5 \\
\hline
\end{tabular}

Tabela 8 - Médias de tipo de desenho por categoria de peso e geral

\begin{tabular}{|c|c|c|c|c|c|c|}
\hline \multirow[t]{2}{*}{ GRUPO } & \multicolumn{2}{|c|}{ ARCO (A) } & \multicolumn{2}{|c|}{ PRESILHA (L) } & \multicolumn{2}{|c|}{ VERTICILO (W) } \\
\hline & $x$ & $S$ & $x$ & $S$ & $x$ & $S$ \\
\hline Ligeiro (-48kg) & 0,3 & $\pm 0,5$ & 6,8 & $\pm 2,9$ & 3,0 & $\pm 3,2$ \\
\hline Meio Leve $(-52 \mathrm{~kg})$ & 0,5 & $\pm 1,0$ & 4,3 & $\pm 4,0$ & 5,3 & $\pm 4,4$ \\
\hline Leve $(-57 \mathrm{~kg})$ & 0,3 & $\pm 0,5$ & 6,5 & $\pm 1,0$ & 3,3 & $\pm 1,3$ \\
\hline Meio Médio (-63kg) & 1,5 & $\pm 2,4$ & 4,8 & $\pm 2,1$ & 3,8 & $\pm 3,3$ \\
\hline Médio $(-70 \mathrm{~kg})$ & 0,5 & $\pm 1,0$ & 5,8 & $\pm 3,9$ & 3,8 & $\pm 4,2$ \\
\hline Meio Pesado (-78kg) & 0,8 & $\pm 1,0$ & 8,0 & $\pm 1,6$ & 1,3 & $\pm 1,9$ \\
\hline Pesado (+78kg) & 0,3 & $\pm 0,5$ & 7,8 & $\pm 1,7$ & 2,0 & $\pm 1,6$ \\
\hline Média Geral & 0,6 & $\pm 1,1$ & 6,3 & $\pm 2,7$ & 3,2 & $\pm 3,0$ \\
\hline
\end{tabular}

Tabela 9 - Médias de D10 e SQTL por categoria de peso e geral

\begin{tabular}{lcccc}
\hline CATEGORIA & \multicolumn{2}{c}{ D10 } & & SQTL \\
\hline & $\mathrm{X}$ & $\mathrm{S}$ & $\mathrm{X}$ & $\mathrm{S}$ \\
Ligeiro (-48kg) & 12,8 & $\pm 3,5$ & 110,8 & $\pm 42,3$ \\
Meio Leve (-52kg) & 14,8 & $\pm 5,0$ & 127,8 & $\pm 54,3$ \\
Leve (-57kg) & 13,0 & $\pm 1,6$ & 11,8 & $\pm 13,2$ \\
Meio Médio (-63kg) & 12,3 & $\pm 5,4$ & 102,0 & $\pm 58,3$ \\
Médio (-70kg) & 13,3 & $\pm 4,7$ & 108,3 & $\pm 24,5$ \\
Meio Pesado (-78kg) & 10,5 & $\pm 2,5$ & 103,0 & $\pm 30,1$ \\
Pesado (+78kg) & 11,8 & $\pm 1,7$ & 100,3 & $\pm 10,7$ \\
Média Geral & 12,6 & $\pm 3,6$ & 109,1 & $\pm 34,4$ \\
\hline
\end{tabular}


Na tabela 6 e gráfico 4, podemos observar os valores médios por grupo e geral para o somatotipo.

Muito poucos estudos foram publicados a respeito do somatotipo de judocas femininas. Na tabela 7 são apresentados alguns destes resultados. Analisando os dados desta tabela, percebemos que os valores encontrados neste estudo revelam diminuição no valor médio de endomorfia, o que pode sugerir que as atletas de alto rendimento do Brasil estão ficando menos endomórficas. Esta mudança seria benéfica para o esporte, uma vez que os melhores resultados alcançados internacionalmente têm uma correlação negativa com a endomorfia.

Apresentamos na tabela 8 as médias de cada tipo de desenho (arco - A; presilha - L; verticilo - W) para cada categoria de peso e a média geral. As figuras 6 e 7 representam graficamente os tipos de desenho das falanges dos dedos por categoria de peso e por grupos, além da média geral.

Se observarmos a tabela 8 e o gráfico 5, com as médias por categoria de peso, a princípio não observamos uma tendência bem definida.

Mas se analisarmos o gráfico 6, onde as categorias são agrupadas duas a duas, observamos claramente que em todos os grupos, bem como na média geral, o percentual de arcos é bem pequeno, não ultrapassando $9 \%$ do total. Este baixo percentual de arcos se repetiu nos estudos de João e Fernandes Filho (2002), Anjos et al. (2003) e Sampaio et al. (2003). É interessante observar que o percentual de presilhas aumenta proporcionalmente ao aumento dos grupos, das categorias mais leves para as mais pesadas, com uma conseqüente diminuição do percentual de verticilos. Esta moderada (indicada pelo valor médio de presilhas), coordenação e resistência de moderada a alta (pelo alto número de verticilos) e resistência de velocidade alta (por ter tanto as presilhas quanto os verticilos em valores médios). Ressaltamos que a resistência de velocidade é muito importante nessas categorias mais leves, pois nelas as lutas costumam ter um ritmo bastante forte durante os cinco minutos de duração. Com relação ao grupo 3, observamos um aumento do número de presilhas e diminuição do percentual de verticilos, o que indicaria uma predisposição deste grupo para um aumento nas manifestações de velocidade e potência e uma resistência de velocidade moderada. No grupo 4, das atletas da categoria pesado, observamos o maior percentual de presilhas de toda a amostra, indicando uma predisposição das atletas desta categoria para um aumento na qualidade de velocidade. A categoria pesado é a categoria mais lenta no judô, no entanto, esta variável pode estar indicando que talvez as atletas que se destacam no alto rendimento precisem ter um componente de velocidade relativamente alto para que possam ter rapidez no momento exato da aplicação da técnica para definir a luta.

Apresentamos, na tabela 9, as médias de delta (D10) e do somatório da qualidade total de linhas (SQTL) para cada categoria de peso e média geral.

Tabela 10 - Contribuição percentual das fórmulas digitais

\begin{tabular}{|c|c|c|c|c|c|c|c|c|}
\hline & $10 \mathrm{~A}$ & $\mathrm{AL}$ & ALW & $10 \mathrm{~L}$ & $\mathrm{~L}=\mathrm{W}$ & $10 \mathrm{~W}$ & $L>W$ & $W>L$ \\
\hline Ligeiro $(-48 \mathrm{~kg})$ & & $25 \%$ & & & & & $50 \%$ & $25 \%$ \\
\hline Meio Leve $(-52 \mathrm{~kg})$ & & & $25 \%$ & & & $25 \%$ & $25 \%$ & $25 \%$ \\
\hline Leve $(-57 \mathrm{~kg})$ & & & $25 \%$ & & $25 \%$ & & $50 \%$ & \\
\hline Meio Médio $(-63 \mathrm{~kg})$ & & $25 \%$ & $25 \%$ & & $25 \%$ & & & $25 \%$ \\
\hline Médio (-70kg) & & & $25 \%$ & & $25 \%$ & $25 \%$ & $25 \%$ & \\
\hline Meio Pesado $(-78 \mathrm{~kg})$ & & $25 \%$ & $25 \%$ & $25 \%$ & & & $25 \%$ & \\
\hline Pesado (+78kg) & & & & $25 \%$ & & & $75 \%$ & \\
\hline Média Geral & $0 \%$ & $10,7 \%$ & $17,9 \%$ & $7,1 \%$ & $10,7 \%$ & $7,1 \%$ & $35,7 \%$ & $10,7 \%$ \\
\hline
\end{tabular}

Tabela 11 - Resultados encontrados por categoria de peso

\begin{tabular}{lccccccccccccc}
\hline CATEG & IDADE & ESTAT & PESO & $\%$ G & ENDO & MESO & ECTO & A & L & W & D10 & SQTL \\
\hline LIG & 23,0 & 157,3 & 52,6 & 18,0 & 2,7 & 3,7 & 2,3 & 0,3 & 6,8 & 3,0 & 12,8 & 110,8 \\
ML & 23,8 & 157,3 & 54,4 & 13,0 & 2,3 & 4,4 & 1,8 & 0,5 & 4,3 & 5,3 & 14,8 & 127,8 \\
LV & 23,0 & 164,3 & 60,8 & 13,7 & 2,2 & 4,2 & 2,0 & 0,3 & 6,5 & 3,3 & 13,0 & 111,8 \\
MM & 23,3 & 163,8 & 63,1 & 17,5 & 2,9 & 4,6 & 1,6 & 1,5 & 4,8 & 3,8 & 12,3 & 102,0 \\
MD & 24,5 & 171,3 & 73,5 & 18,6 & 4,0 & 5,0 & 1,4 & 0,5 & 5,8 & 3,8 & 13,3 & 108,3 \\
MP & 23,5 & 171,8 & 78,0 & 22,4 & 3,9 & 5,3 & 1,0 & 0,8 & 8,0 & 1,3 & 10,5 & 103,0 \\
PES & 22,3 & 168,3 & 103,3 & 37,8 & 7,0 & 8,6 & 0,1 & 0,3 & 7,8 & 2,0 & 11,8 & 100,3 \\
MÉDIA & 23,3 & 164,8 & 69,4 & 20,1 & 3,6 & 5,1 & 1,5 & 0,6 & 6,3 & 3,2 & 12,6 & 109,1 \\
\hline
\end{tabular}

Tabela 12 - Resultados encontrados por categoria de peso

\begin{tabular}{|c|c|c|c|c|c|c|c|c|c|c|c|c|}
\hline CATEG & IDADE & ESTAT & PESO & $\% \mathrm{G}$ & ENDO & MESO & ЕСTO & A & L & W & D10 & SQTL \\
\hline GR 1 & 23,4 & 157,3 & 53,5 & 15,5 & 2,5 & 4,1 & 2,1 & 0,4 & 5,5 & 4,1 & 13,8 & 119,3 \\
\hline GR 2 & 23,1 & 164,0 & 61,9 & 15,6 & 2,6 & 4,4 & 1,8 & 0,9 & 5,6 & 3,5 & 12,6 & 106,9 \\
\hline GR 3 & 23,9 & 171,5 & 75,7 & 20,5 & 4,0 & 5,2 & 1,2 & 0,6 & 6,9 & 2,5 & 11,9 & 105,6 \\
\hline GR 4 & 22,3 & 168,3 & 103,3 & 37,8 & 7,0 & 8,6 & 0,1 & 0,3 & 7,8 & 2,0 & 11,8 & 100,3 \\
\hline MÉDIA & 23,3 & 164,8 & 69,4 & 20,1 & 3,6 & 5,1 & 1,5 & 0,6 & 6,3 & 3,2 & 12,6 & 109,1 \\
\hline
\end{tabular}


Se observarmos a tabela 9 e o gráfico 7 contendo os valores médios de D10 e SQTL para cada categoria de peso, novamente não observamos de imediato uma tendência bem definida. Mas ao analisarmos o gráfico 8 , com as médias destes valores para os grupos, observamos uma tendência bastante definida de diminuição, tanto de D10 quanto de SQTL, conforme o aumento dos pesos das categorias com os grupos. No grupo 4, confirma-se o aumento para predisposição à velocidade, pois segundo Guba e Tchernova (1995), citados por FERNANDES FILHO (1997), o aumento da quantidade de linhas é contrário ao desenvolvimento das qualidades de velocidade e força, conseqüentemente de potência. Já o grupo 1, das atletas mais leves, tem tanto D10 quanto SQTL aproximadamente $20 \%$ maior do que a média do grupo 4 . Segundo Fernandes Filho (1997), esta elevação nos níveis de D10 e SQTL se correlaciona com o aumento das dominantes de resistência e coordenação.

Os gráficos 7 e 8 representam o D10 e o SQTL por categoria de peso e por grupos, além da média geral.

Podemos encontrar oito tipos de fórmulas digitais. Apresentamos na tabela 10 as médias encontradas por categoria de peso e geral.

Como podemos perceber, para esta amostra de atletas de alto rendimento do judô feminino brasileiro, o maior percentual $(35,7 \%)$ de atletas possui a fórmula digital $L>W$. Se somarmos a este percentual os outros percentuais que envolvem apenas presilhas e verticilos $(W>L=10,7 \%, L=W=10,7 \%)$, teremos $57,1 \%$ da amostra. Apenas 28,6\% da amostra, em média, possuem o desenho arco em pelo menos um dos dedos (AL $=10,7 \%$ e $\mathrm{ALW}=17,9 \%$ ). Estes dados demonstram uma tendência para atletas com maior percentual de fibras rápidas no judô feminino de alto rendimento no Brasil.

O gráfico 9 representa graficamente a contribuição percentual de cada tipo de fórmula digital na média desta amostra.

gráfico 10 apresenta os dados normatizados para o tipo de desenho, o índice de delta (D10) e o somatório da quantidade total de linhas nos dez dedos das mãos (SQTL).

\section{CONCLUSÕES}

As tabelas 11 e 12 apresentam, respectivamente, os resultados do perfil, por categoria de peso e por grupos, nas variáveis estudadas.

Quanto à composição corporal, a média da categoria pesado foi de $37,8 \%$, valor bastante elevado para atletas de alto rendimento. Se excluíssemos desta amostra a categoria de peso pesado, que não tem limite de peso e tem um percentual de gordura bem mais elevado que a média, teríamos uma média para as outras categorias de peso de 17,2\%. Concluímos que, de uma forma geral, as categorias ligeiro a meio pesado têm um percentual de gordura médio comparável ao de atletas de alto rendimento de outros países. Analisando a categoria ligeiro $(-48 \mathrm{~kg})$, a categoria de peso mais leve no judô, percebemos que estas atletas tiveram média de percentual de gordura de 18,0\%, o que é bastante elevado para a categoria. Este percentual foi maior do que as três categorias superiores, quase se igualando ao percentual de gordura da categoria médio $(-70 \mathrm{~kg})$. Tal valor elevado sugere que, provavelmente, as atletas da categoria ligeiro não estavam seguindo uma dieta alimentar adequada durante o período de treinamento, o que possivelmente terá reflexos negativos em seus desempenhos competitivos.

De acordo com os resultados apresentados para o somatotipo (média de 3,6-5,1-1,5), podemos concluir que o somatotipo ideal para atletas de judô feminino de alto rendimento adultas é aquele com predominância da mesomorfia, em todas as categorias de peso, mesmo na categoria pesado. Se excluíssemos a categoria pesado, que tem um somatotipo bastante distante da média (SAMGr4,MD = 17,61), teríamos um somatotipo médio de $3,0-4,5-1,7$.

Quanto às características dermatoglíficas, obtivemos para esta amostra uma média de 0,6 $\operatorname{arcos}(A), 6,3$ presilhas $(L)$ e 3,2 verticilos (W). A partir destes valores, e com base nas referências apresentadas anteriormente, podemos concluir que esta amostra de judocas brasileiras de alto rendimento tem uma predominância genética de fibras musculares rápidas. Ao analisarmos os grupos, percebemos uma clara tendência de aumento gradual de presilhas e diminuição de verticilos com o aumento do peso, o que conforme Fernandes Filho (2003), direciona para uma tendência de aumento nas qualidades físicas de velocidade e força explosiva. Este fato talvez se explique pela necessidade de uma maior velocidade em um momento específico durante a luta, necessário para as atletas mais pesadas, que tem como característica das categorias de peso uma luta mais lenta.

Com relação ao índice de delta (D10) e ao somatório da quantidade total de linhas nos dez dedos das mãos (SQTL), foi encontrada uma média de 12,6 para D10 e 109,1 para SQTL. No grupo 4, confirma-se o aumento para predisposição à velocidade, pois o aumento da quantidade de linhas é contrário ao desenvolvimento das qualidades de velocidade e força, conseqüentemente de potência. Já o grupo 1, das atletas mais leves, tem tanto D10 quanto SQTL aproximadamente 20\% maior do que a média do grupo 4. Segundo Fernandes Filho (1997), esta elevação nos níveis de D10 e SQTL se correlaciona com o aumento das dominantes de resistência e coordenação. A partir dos dados apresentados, podemos concluir que o judô feminino de alto rendimento é um esporte caracterizado por atletas com maior predisposição para desenvolvimento das capacidades de velocidade e força.

\section{REFERÊNCIAS}

ANJOS, M. A. B . FERNANDES FILHO, J. NOVAES, J S Características somatotípicas, dermatoglíficas e fisiológicas do atleta de triatlo. Fitness \& Performance Journal, v.02, n.01, 2003.

BEIGUELMAN, B. Dinâmica dos Genes nas Famílias e nas Populações. $2^{a}$ ed. Ribeirão Preto: Revista Brasileira de Genética, 1995.

CARTER, J. E. L.; HEATH, B. H. Somatotyping Development and Applications. New York: Cambridge University Press, 1990, 503p.

CARUSO, P.R.; GROSSO, F.; ABRANTES, R.J.; DANTAS, P.; FERNANDES FILHO, J. Identificação dos Perfis Dermatoglífico e Somatotípico de Lutadores de Vale Tudo da Cidade do Rio de Janeiro). Rio de Janeiro, [2003]. Disponível em: <http://www.portaldovaletudo. com.br/idesomatotipo.htm> Acesso em: 02 jan 2004 
CARVALHO, E. Identificação do perfil dermatoglífico, somatotípico e fisiológico que Caracterizam Atletas Masculinos de Alto Rendimento participantes de corridas de resistência no Rio de Janeiro. Dissertação de mestrado em Ciência da Motricidade Humana. Rio de Janeiro: Universidade Castelo Branco, 2003.

CASTANHEDE, A. L. K.; DANTAS, P. M. S.; FERNANDES FILHO, J. Perfil dermatoglíico, e somatotípico de atletas de futebol de campo masculino, de alto rendimento no Rio de Janeiro - BRASIL. Fitness \& Performance Journal, v.02, n.04, 2003.

CUMMINS H.; MIDLO CH. Palmar and plantar dermatoglyphics in primates. Philadelphia, 1942.

DANTAS, P. M. S. Identificação dos Perfis Genéticos, de Aptidão Física e Somatotípico que Caracterizam Atletas Masculino de Alto Rendimento participantes do Futsal Adulto no Brasil. Dissertação de mestrado em Ciência da Motricidade Humana. Rio de Janeiro: Universidade Castelo Branco, 2001, 208p.

FERNANDES FILHO, J. Impressões dermatoglíficas - marcas genéticas na seleção dos tipos de esporte e lutas (a exemplo de desportista do Brasil). Tese de Doutorado, Moscou URSS, 1997.

. A prática da avaliação física. 2. ed. Rio de Janeiro: Shape, 2003.

. Descoberta de talentos. 1. ed. Rio de Janeiro: Shape, v.1, n.2, versão 1.1, 2003a, CD-ROM.

FERNANDES FILHO, J.; ROQUETTI FERNANDES, P.; DANTAS, P. M. S. Dermatoglifia $x$ Diagnóstico. Fitness \& Performance Journal, v.03, n.01, 2004.

FRANCHINI, E. Bases para detecção e promoção de talentos na modalidade judô. In Instituto Nacional de Desenvolvimento do Desporto (INDESP). Prêmio INDESP de literaturo desportiva. Brasilia: INDESP, 1999. p. 17-104.

FRANCHINI, E. Judô - Desempenho Competitivo. Barueri: Editora Manole, 2001.

GRECO, P. J.; VIANA, J. M. Os Princípios do Treinamento Técnico Aplicados ao Judô e a inter-relação com as fases do Treinamento. Revista de Educação Física / UEM, Maringá Paraná, v.8, n.1, p.37-43, 1997.

JOÃO, A.; FERNANDES FILHO, J. Identificação do perfil genético, somatotípico e psicológico das atletas brasileiras de ginástica olímpica feminina de alta qualificação esportiva. Fitness \& Performance Journal, v.01, n.02, 2002.
MACÊDO. M. M.; FERNANDES FILHO, J. Estudo das características dermatoglíficas, somatotípicas e das qualidades físicas básicas nos diversos estágios de maturação sexual. Fitness \& Performance Journal, v.2, n.6, p.315-320, nov/dez, 2003

MEDINA, M. F. Identificação dos perfis genético e somatotípico que caracterizam atletas de voleibol masculino adulto de alto rendimento no Brasil. Dissertação de mestrado em Ciência da Motricidade Humana. Rio de Janeiro: Universidade Castelo Branco, 2001.

MELLO, M. V.; MORAES, J. M.; FERNANDES FILHO, J. Composição corporal e somatotipo das atletas da seleção de judô feminino do Rio de Janeiro participantes do Campeonato Brasileiro Feminino Sênior de 1999. Revista de Educação Física, n. 125, pp.29-35, 2001.

NORTON, K.; OLDS, T. Anthropometrica. Sydney: University of New South Wales Press, 1996, $411 p$

PÁVEL, D. A. C. Identificação dos perfis dermatoglífico, somatotípico e de qualidades físicas básicas de atletas de alto rendimento na modalidade de natação em provas de meio-fundo e fundo. Dissertação de mestrado em Ciência da Motricidade Humana. Rio de Janeiro: Universidade Castelo Branco, 2003.

SAMPAIO, A. O.; DANTAS, P. M. S.; FAZOLO, E.; FERNANDES FILHO, J. Perfis somatotípico, de qualidades físicas básicas e dermatoglífico dos pilotos de caça da F.A.B. com as patentes de Tenente e Capitão. Fitness \& Performance Journal, v.02, n.02, 2003.

SILVA, E.; FREITAS, W.; FERRÃO, M. L.; FERNANDES FILHO, J. DANTAS, E. H. Níveis de flexibilidade em função do tipo de fibra muscular. Fitness \& Performance Journal, v.02, n.03, 2003.

SIRI, W. E. Body composition from fluid space and density. In: BROZEK, J.; HANSCHEL, A. (Eds). Techniques for measuring body composition. Washington: National Academy of Science, 1961

SOCIEDADE INTERNACIONAL PARA AVANÇO DA CINEANTROPOMETRIA (ISAK). Apostilo de curso. Rio de Janeiro, 2000.

SUGAI , V. L.; colaboração de TSUIMOTO, S. O Caminho do Guerreiro I. São Paulo: Editora Gente, 2000, 229p. 